\title{
Asociación y relación predictiva del distrés con variables sociodemográficas y clínicas en cáncer mamario
}

\section{Association and predictive relationship between distress and sociodemographic and clinical variables in breast cancer}

José Moral de la Rubia

Melina Miaja Ávila

Universidad Autónoma de Nuevo León

\section{Resumen}

El distrés es un estado emocional negativo que puede ser inducido por enfermedades crónicas como el cáncer. Para su medición existe un instrumento con buenas propiedades métricas como el Inventario Breve de Síntomas. Su forma abreviada a 18 ítems (BSI-18) ha sido validada en aspectos de estructura factorial y consistencia interna en México, pero no en aspectos de validez concurrente. En la práctica clínica con mujeres con cáncer de mama, es importante identificar las variables que predicen mayor distrés para enfocar la atención psicológica. A este fin se puede aplicar el BSI-18 con la nueva estructura factorial identificada en México. Esta investigación tuvo como objetivo estudiar la relación del distrés con variables sociodemográficas y clínicas. Se utilizó un muestreo intencional. La muestra quedó integrada por 203 mujeres mexicanas tratadas por cáncer de mama. Se aplicó un cuestionario de autorreporte integrado por preguntas cerradas sobre información sociodemográfica y clínica y el BSI-18. La puntuación total y los tres factores del BSI-18 fueron independientes de las variables sociodemográficas. En la muestra total, recibir terapia antihormonal y recibir radioterapia destacaron como correlatos y predictores entre las variables clínicas. Entre las mujeres mastectomizadas, recibir quimioterapia y desear conservar la mama fueron los correlatos y predictores más importantes. Mayores períodos de tiempo transcurrido desde el diagnóstico y desde la cirugía se asociaron a más distrés. Se concluye que proporcionar apoyo psicológico a las mujeres que reciben terapia antihormonal y radioterapia es importante, especialmente considerando que a más tiempo de tratamiento se incrementa el distrés.

Palabras clave: distrés, cáncer, mastectomía, deseo de conservar la mama, quimioterapia.

Nota del autor

José Moral de la Rubia, Facultad de Psicología, Universidad Autónoma de Nuevo León. Melina Miaja Ávila, Facultad de Psicología, Universidad Autónoma de Nuevo León.

La correspondencia en relación con este artículo debe dirigirse a José Moral de la Rubia, Universidad Autónoma de Nuevo León, c/Mutualismo 110, C.P. 64640, Monterrey, Nuevo León, México.

Correo electrónico: jose_moral@hotmail.com 


\begin{abstract}
Distress is a negative emotional state that can be induced by chronic diseases such as cancer. For its measurement, the Brief Symptom Inventory is an instrument with adequate metric properties. Its abbreviated form (to 18 items (BSI-18)) has been validated in aspects of factorial structure and internal consistency in Mexico but not in aspects of concurrent validity. In clinical practice with women with breast cancer, it is important to identify the variables that predict greater distress in order to address psychological wellbeing. To this aim, the BSI-18 can be applied with the new identified factorial structure in Mexico. The objective of this research was to study the relationship between distress and sociodemographic and clinical variables. An intentional, non-probabilistic sampling method was used. The sample was composed of 203 Mexican women treated for breast cancer. The BSI18 and a self-report questionnaire were administered. The latter consisted of close-ended questions regarding sociodemographic and clinical information. The BSI-18 total score and its three factors were independent of the sociodemographic variables. In the total sample, receiving anti-hormone therapy and receiving radiotherapy were highlighted as correlates and predictors among the clinical variables. In mastectomized women, receiving chemotherapy and desiring to conserve the breast were the most important correlates and predictors. Longer periods of time elapsed since diagnosis and surgery were associated with greater distress. We conclude that providing psychological support to women receiving anti-hormone therapy and radiotherapy is important, especially considering that longer treatment times increase levels of distress.
\end{abstract}

Keywords: distress, cancer, mastectomy, desire to preserve the breast, chemotherapy.

Se define distrés como un estado de malestar emocional con síntomas de ansiedad y depresión (Holland et al., 2010). El distrés tiene un impacto como factor etiológico, agravante o cronificador en diversas enfermedades funcionales, como el síndrome de intestino irritable (Enríquez, Rodríguez, \& Schneider, 2010), la fibromialgia (Wolfe, Brähler, Hinz, \& Häuser, 2013) y la hipertensión esencial (Ojike et al., 2016), así como en enfermedades somáticas, como el cáncer (Segrin \& Badger, 2014). El instrumento más usado para su medición es el Inventario Breve de Síntomas con 18 ítems, el BSI-18 (Hernández et al. 2012), el cual permite evaluar los factores de somatización, depresión y ansiedad (Derogatis, 2001).
Kagan y Levi (1975) propusieron un modelo teórico para conceptualizar el distrés, al que denominaron modelo de mediación psicosocial de la enfermedad. Este modelo postula que los estímulos ambientales cargados de significado y valencia afectiva activan programas biológicos de respuesta, como puede ser la activación del eje del estrés hipotalámico-hipofisario-adrenal. Estos programas biológicos están mediados por influencias ambientales tempranas $y$ factores genéticos. Los significados dados a los eventos de interacción social y las respuestas fisiológicas percibidas activan mecanismos de afrontamiento que pueden resolver el estado de intensa activación fisiológica. No obstante, la persistencia de una activación fuerte del 
eje hipotalámico-hipofisario-adrenal genera un desbalance vegetativo que se expresa en síntomas somáticos y malestar subjetivo. La medición del estrés a través del BSI se centra en estas consecuencias.

El BSI-18 ha sido estudiado en México: Miaja y Moral (2017), en una muestra de 203 mujeres diagnosticadas con cáncer, hallaron que el modelo con mejor ajuste era uno de tres factores jerarquizados a un factor general de distrés. Los tres factores de segundo orden se asemejaron a los definidos por Derogatis (2001). El factor de depresión quedó definido por los mismos seis ítems. El factor de somatización perdió el ítem 4 y en su lugar apareció el ítem 15, lo que varió su contenido y fue denominado síntomas vasovagales de ansiedad (desmayo, náuseas, falta de aire, debilidad, adormecimiento e inquietud). El factor de ansiedad perdió el ítem 15 y en su lugar apareció el ítem 4. Al nuevo contenido se le denominó síntomas simpáticos de ansiedad (nerviosismo, temblor, tensión, sobresaltos, ataques de pánico, miedo y dolores en el pecho). Este nuevo modelo de ansiedad y depresión matiza entre dos expresiones de la ansiedad, una ligada a preocupaciones hipocóndricas y otra a situaciones de ataque/huida ante peligros (Owens, Low, Iodice, Critchley, \& Mathis, 2017; Simon, Meuret, \& Ritz, 2017).

Se ha estudiado la relación del distrés, medido con el BSI-18, con variables sociodemográficas y clínicas. Un menor malestar emocional se asocia a una mayor edad (Andreu et al., 2012; Budden, Hayes, \& Buettner, 2014; Carlson et al., 2004; Champion et al., 2014), un mayor grado de escolaridad (Miaja \& Moral, 2014), un mayor nivel de ingresos económicos (Carlson et al., 2004) y estar casada (Budden et al., 2014; Fávez et al., 2015). A su vez, un menor malestar emocional se asocia a un intervalo más largo de tiempo transcurrido desde el diagnóstico (Andreu et al., 2012), un estadio menos avanzado del cáncer, no tener comorbilidad de diabetes o hipertensión (Holland et al., 2010), no estar en tratamiento activo (Carlson et al., 2004), no haber recibido quimioterapia (Hjörleifsdóttir, Hallberg, Bolmsjö, \& Gunnarsdóttir, 2006) y no haber sufrido una intervención de mastectomía (Reich, Lesur, \& Perdrizet-Chevallier, 2008).

Considerando el modelo factorial definido por Miaja y Moral (2017) para el BSI-18, el primer objetivo de la presente investigación es analizar la asociación de dicho instrumento con cuatro variables sociodemográficas (edad, escolaridad, ingresos económicos y estado civil) y cinco variables clínicas (tiempo transcurrido desde el diagnóstico, tiempo transcurrido desde la cirugía, estadio del cáncer, comorbilidad y tipo de intervención quirúrgica). Con este objetivo se pretende aportar evidencias de validez concurrente para el BSI-18 como un instrumento que mide distrés. El segundo objetivo es generar modelos de predicción para la puntuación total y los tres factores del BSI-18 con las variables previamente mencionadas.

Se espera que las variables clínicas sean correlatos y predictores del BSI-18 y sus tres factores (Hjörleifsdóttir et al., 2006; Reich et al., 2008), teniendo menor efecto las variables sociodemográficas; entre estas se espera que destaque la edad, asociándose menor edad a más distrés (Champion et al., 2014). La expectativa 
es que el tipo de tratamiento oncológico recibido (por ejemplo terapia antihormonal) destaque entre las variables clínicas, actuando como factor de riesgo por sus efectos secundarios (Carlson et al., 2004). Debido al hecho de que las mujeres están en tratamiento activo con una variabilidad de años, se espera que el tiempo transcurrido desde el diagnóstico y el transcurrido desde la cirugía actúen como factores de riesgo, al implicar mayor cronicidad o recidivas (Andreu et al., 2012).

\section{Método}

Se realizó un estudio empírico con metodología cuantitativa con diseño ex post facto transversal.

\section{Participantes}

Los criterios de inclusión fueron: ser mujer con diagnóstico de cáncer de mama, estar recibiendo tratamiento oncológico en el Hospital San José (hospital privado) y estar afiliada al Seguro Popular. El Seguro Popular puede ser percibido por quienes no sean beneficiarios de ninguna otra institución de seguridad social. Procede de un fondo de protección contra gastos catastróficos de carácter federal. Proporciona recursos monetarios a través de un fideicomiso a los prestadores de servicios acreditados y con convenios firmados (Comisión Nacional de Protección Social en Salud, 2019). El hospital San José está suscrito a este programa para la atención del cáncer de mama. Como criterios de exclusión se fijaron: déficit cognitivo que impidiese comprender las instrucciones, fatiga excesiva que dificultase la atención y comprensión de las preguntas.
Se utilizó un muestreo no probabilístico intencional; la muestra recolectada fue de 203 mujeres. La media de edad fue 48.23 años con una desviación estándar de 9.87 y un rango de 30 a 79 . Con respecto al estado civil, 61.6\% estaban casadas, $12.3 \%$ vivían en unión libre con la pareja, $10.8 \%$ estaban solteras, 9.9\% separadas o divorciadas y $5.4 \%$ viudas. El $64 \%$ indicaron ser amas de casa, 19.7\% trabajadoras laboralmente activas, 7.9\% desempleadas, 5.4\% con incapacidad laboral médica, $2.5 \%$ jubiladas y $0.5 \%$ estudiantes. La mediana de escolaridad correspondió a estudios de media superior; el $0.5 \%$ no tuvo ningún estudio; $21.7 \%$ tuvo primaria; $26.6 \%$, secundaria; $22.7 \%$, media superior; $28.1 \%$, licenciatura, y $0.5 \%$, posgrado. La mediana de ingreso económico familiar mensual correspondió al intervalo de 3000 a 5 999 pesos mexicanos (aproximadamente entre 161 y 321 dólares estadounidenses); el 29.6\% reportó menos de 3000 pesos ( $<161$ dólares); $38.2 \%$, entre 3000 y 5999 ( $\approx$ entre 161 y 321 dólares); 19.6\%, entre 6000 y $11999(\approx$ entre 321 y 642 dólares); 9.5\%, entre 12000 y 23999 ( $\approx$ entre 642 y 1,283 dólares ), y $3 \%$, entre 24000 y 47999 pesos ( $\approx$ entre 1,283 y 2,638 dólares). En la Tabla 1 puede encontrarse la descripción de las variables clínicas. Cabe señalar que los datos analizados en la presente investigación proceden de la misma muestra que se usó en el estudio de Miaja y Moral (2017) sobre la estructura factorial y consistencia interna del BSI-18; no obstante, el planteamiento, los datos analizados y discutidos son distintos en ambos estudios. 
Tabla 1

Distribuciones de las variables sociodemográficas y clínicas

\begin{tabular}{|c|c|c|c|c|c|c|}
\hline \multicolumn{3}{|l|}{ Variable clínica } & \multicolumn{2}{|l|}{$N$} & \multicolumn{2}{|l|}{$\%$} \\
\hline \multirow{2}{*}{ Comorbilidad } & Sí & & 53 & & 27.7 & \\
\hline & No & & 138 & & 72.3 & \\
\hline \multirow{2}{*}{ Hipertensión arterial } & Sí & & 38 & & 19.9 & \\
\hline & No & & 153 & & 80.1 & \\
\hline \multirow{2}{*}{ Diabetes } & Sí & & 33 & & 17.3 & \\
\hline & No & & 158 & & 82.7 & \\
\hline \multirow{2}{*}{$\begin{array}{l}\text { Antecedentes } \\
\text { familiares de cáncer }\end{array}$} & Sí & & 108 & & 56.5 & \\
\hline & No & & 83 & & 43.5 & \\
\hline \multirow{2}{*}{$\begin{array}{l}\text { Antecedentes } \\
\text { personales de cáncer }\end{array}$} & Sí & & 15 & & 7.9 & \\
\hline & No & & 176 & & 92.1 & \\
\hline \multirow{3}{*}{ Triple negativo en RE, RP y HER2 } & Sí & & 51 & & 26.7 & \\
\hline & No & & 140 & & 73.3 & \\
\hline & 0 & & 1 & & 0.5 & \\
\hline \multirow{7}{*}{ Estadio del cáncer } & $1 \mathrm{~A}$ & & 13 & & 6.8 & \\
\hline & 1B & & 2 & & 1.0 & \\
\hline & $2 \mathrm{~A}$ & & 43 & & 22.5 & \\
\hline & $2 \mathrm{~B}$ & & 43 & & 22.5 & \\
\hline & $3 A$ & & 45 & & 23.6 & \\
\hline & 3B & & 14 & & 7.3 & \\
\hline & $3 C$ & & 10 & & 5.2 & \\
\hline \multirow{4}{*}{ Cirugía practicada } & 4 & & 20 & & 10.5 & \\
\hline & Conservadora & & 46 & & 23.6 & \\
\hline & \multirow[b]{2}{*}{ Mastectomía } & DCM & \multirow[b]{2}{*}{69} & 33 & \multirow{2}{*}{35.4} & 49.3 \\
\hline & & NDCM & & 34 & & 50.7 \\
\hline No & Pendiente & & 80 & & 41.0 & \\
\hline \multirow{2}{*}{ Quimioterapia } & $\mathrm{Si}$ & & 175 & & 91.6 & \\
\hline & No & & 16 & & 8.4 & \\
\hline \multirow{2}{*}{ Radioterapia } & Sí & & 36 & & 18.8 & \\
\hline & No & & 155 & & 81.2 & \\
\hline \multirow{2}{*}{ Inmunoterapia } & Sí & & 16 & & 8.4 & \\
\hline & No & & 175 & & 91.6 & \\
\hline \multirow{2}{*}{ Terapia antihormonal } & Sí & & 32 & & 16.8 & \\
\hline & No & & 159 & & 83.2 & \\
\hline
\end{tabular}

Nota. $n=$ frecuencia absoluta simple, $\%$ porcentaje. $\mathrm{RE}=$ receptores de estrógeno, $\mathrm{RP}=$ receptores de progesterona y HER2 = receptor 2 del factor de crecimiento epidérmico humano. DCM = Deseaba conservar la mama, NDCM = No deseaba conservar la mama. Fuente: elaborada por los autores. 


\section{Instrumentos de medida}

Se aplicó un cuestionario integrado por preguntas cerradas sobre datos sociodemográficos y clínicos y el BSI-18 con la traducción hecha en España (Derogatis, 2013). El BSI-18 es una escala de autoinforme que mide la frecuencia con la que se ha experimentado síntomas somáticos y emocionales en los últimos siete días. Está conformado por 18 ítems directos con cinco opciones de respuesta (de $0=$ "nada" a 4 = “mucho”).

En su estudio original (Derogatis, 2001), el BSI-18 presentó consistencia interna buena ( $\alpha$ de Cronbach $=.89$ ) y una estructura de tres factores: somatización, depresión y ansiedad. En México con sus 18 ítems, se definieron tres factores, aunque con una composición distinta a la original: síntomas de depresión (ítems 2, 5, 8, 11,14 y 17) con consistencia interna buena $(\alpha=$ .84 ), síntomas simpáticos de ansiedad (ítems 3 , $4,6,9,12$ y 18$)$ con consistencia aceptable $(\alpha=$ .78) y síntomas vasovagales de ansiedad (ítems $1,7,10,13,15$ y 16) también con consistencia interna aceptable $(\alpha=.72)$. La consistencia de los 18 ítems fue buena $(\alpha=.89)$ (Miaja \& Moral, 2017). Las puntuaciones en el BSI-18 y sus tres factores se obtuvieron sumando los ítems y dividiendo por el número de ítems sumados, con lo que resulta un rango continuo de 0 a 4 . Los factores fueron definidos desde su configuración en la adaptación de Miaja y Moral (2017).

\section{Procedimientos}

Las participantes fueron invitadas a la investigación por estudiantes de la Licenciatura en Psicología Clínica y de la Salud y por la segunda autora del artículo. A estos estudiantes se les dio una breve capacitación para este fin. Al momento de invitar a las pacientes oncológicas se les daba información sobre la investigación y se les explicaba en qué consistiría su participación. Solo se administró el cuestionario a aquellas que firmaron voluntariamente la carta de consentimiento informado, donde se garantizaba la confidencialidad de los datos, de acuerdo a las normas de investigación de la American Psychological Association (2017).

Todas las mujeres invitadas dieron el consentimiento; no obstante, 30 de ellas no pudieron terminar de responder el cuestionario por fatiga, por lo que no fueron integradas en la muestra. Respondieron por escrito al cuestionario en cuartos de infusión y salas de espera del Centro de Cáncer de Mama del Tecnológico de Monterrey. En estos cubículos, se encontraban varias pacientes y la psicóloga antes mencionada o un alumno colaborador, quien entregaba y recogía el cuestionario $\mathrm{y}$ a quien se podía preguntar en caso de dudas. El estudio fue aprobado por las Comisiones de Ética y de Investigación de la Escuela de Medicina del Tecnológico de Monterrey y de la Fundación Santos y de la Garza Evia I.B.P. La muestra se recolectó de agosto de 2016 a mayo de 2017.

\section{Análisis de datos}

Por una parte, se estudió la relación de las puntuaciones en el BSI-18 y sus tres factores con las variables sociodemográficas y clínicas 
por medio de correlaciones y comparación de medias aritméticas. Por otra parte, se generaron modelos predictivos para estas puntuaciones en el BSI-18 y sus tres factores a través de regresión logística binaria.

Las correlaciones de las puntuaciones en el BSI y sus tres factores con las variables numéricas (edad, tiempo transcurrido desde el diagnóstico y cirugía) se calcularon por el coeficiente de correlación producto-momento de Pearson $(r)$ y con las variables ordinales (escolaridad e ingreso económico) por el coeficiente de rangos de Spearman $\left(r_{S}\right)$. Un valor de $r$ o $r_{S}$ entre $-.09 \mathrm{y}$ .09 se interpretó como una fuerza de asociación trivial; entre -.29 y -.10 o .10 y .29 , baja; entre -.49 y -.30 о .30 y .49 , moderada; entre -.69 y -.50 o .50 a .69 , alta; entre -.89 y -.70 o .70 a .89 muy alta, y entre -1 y -.90 o .90 y 1 , unitaria (Cohen, 1988).

Se compararon las medias aritméticas en las puntuaciones en el BSI y sus tres factores entre los grupos de estado civil, situación laboral, estadio del cáncer e intervención quirúrgica (en espera, conservadora y mastectomía) por análisis de varianza (ANOVA). Las comparaciones de medias entre mujeres con o sin mastectomía, con o sin diabetes y con o sin hipertensión se hicieron por la prueba $\mathrm{t}$ de Student para dos muestras independientes. En el caso del ANOVA, el tamaño del efecto se calculó con el coeficiente eta cuadrado. En el caso de la t de Student, se estimó por la g de Hedges y Olkin corregida de sesgo. Se interpretó que valores de $g<0.20$ reflejan un tamaño del efecto trivial; de
0.20 a 0.49 , pequeño; de 0.50 a 0.79 , grande; de 0.80 a 1.20 , muy grande, $\mathrm{y} \geq 2$, inmenso (Hedges \& Olkin, 1985; Sawilowsky, 2009).

Debido a la heterogeneidad de la muestra en la variable de tiempo transcurrido desde el diagnóstico, esta variable numérica se controló estadísticamente para comprobar la sustantividad del efecto de las variables clínicas sobre el BSI$18 \mathrm{y}$ sus factores. No fue necesario con las variables sociodemográficas, porque estas no tuvieron efecto. Se usó análisis de covarianza (ANCOVA) con las variables cualitativas, y se utilizó la correlación parcial de Fisher con la variable numérica: tiempo transcurrido desde la cirugía. Adicionalmente, esta última variable numérica se controló estadísticamente para comprobar la sustantividad del efecto del deseo de conservar la mama sobre el BSI-18 y sus tres factores, usando análisis de covarianza.

Para calcular los modelos de regresión logística binaria, se dicotomizaron las puntuaciones en el BSI-18 y sus tres factores. Por una parte, se usó el percentil 75 (caso $\geq$ P75) como sugieren Zabora et al. (2001). Por otra parte, se usó el percentil 90 (caso $\geq$ P90) como sugiere Derogatis (2001). Estas medidas de posición se calcularon con los datos muestrales, ya que los estudios de Derogatis (2001) y Zabora et al. (2001) fueron hechos con muestras estadounidenses. En la muestra total y en la submuestra de mujeres con mastectomía, se identificaron los correlatos de estas variables dicotomizadas, usando la correlación biserial con las variables numéricas y la prueba chi- 
cuadrado con la corrección de Yates con las variables dicotómicas. Luego, se calcularon los modelos por el método de pasos progresivos hacia adelante con el criterio de probabilidad condicional con los correlatos significativos.

El tamaño del efecto de las variables predictoras en los modelos de regresión logística binaria se estimó a través de la razón de probabilidades u oportunidad relativa $(O R)$. Al transformar el $O R$ en un estadístico $d$ a través de la fórmula de Chinn (2000) y considerar los puntos de corte propuestos por Cohen (1988) y Sawilowsky (2009), se interpretó que un valor de $O R$ entre 1 y 1.43 refleja un tamaño del efecto trivial; de 1.44 a 2.46, pequeño; de 2.47 a 4.24 , medio; de 4.25 a 8.76 , grande; de 8.77 a 37.33 , muy grande, $\mathrm{y} \geq 37.34$, inmenso.

Se contrastó la normalidad univariada en las distribuciones de las variables cuantitativas (puntuaciones en BSI-18 y sus tres factores, edad y tiempo transcurrido desde el diagnóstico y la cirugía) por la prueba de Kolmogorov y Smirnov con la corrección de Lilliefors. A su vez, se comprobó la normalidad bivariada en el contraste de la significación de las correlaciones por el estadístico $U$ de asimetría multivariada y el estadístico $\mathrm{W}$ de curtosis multivariada (Kankainen, Taskinen, \& Oja, 2007). Solo se mantuvo el supuesto de normalidad univariada con la edad: $\operatorname{Max}[D(203)]=0.061, p=.062$. En todos los demás casos, se incumplió.

Salvo en las comparaciones de medias en edad, los errores estándar e intervalos de confianza de las correlaciones y diferencias medias se calcularon por el método de muestreo repetitivo corregido de sesgo y acelerado con la simulación de 1000 muestras. La no inclusión del cero en los intervalos (corregidos de sesgo y acelerados) calculados con un nivel de significación al 95\% se requirió como confirmación de los resultados de los contrastes que asumían normalidad (prueba $\mathrm{t}$ para comparar medias y probar la significación de las correlaciones, ANOVA y ANCOVA).

Los contrastes fueron bilaterales con un nivel de significación de .05. Los cálculos fueron hechos con el programa SPSS versión 24, módulo R 4.2 para SPSS24 y Excel 2013.

\section{Resultados}

\section{Relación con variables sociodemográficas y clínicas}

Las puntuaciones en el BSI-18 y sus tres factores fueron independientes de las cinco variables sociodemográficas. Al comparar las medias en la puntuación total del BSI-18 y sus tres factores entre los grupos con o sin comorbilidad, con o sin diabetes, con o sin hipertensión, con o sin antecedentes familiares de cáncer, con o sin antecedentes personales de cáncer y con o sin perfil triple negativo (en receptores de estrógenos, RE, receptores de progesterona, RP, y proteína HER2/neu), solo se halló diferencia significativa en diabetes comórbida. La media en síntomas simpáticos de ansiedad de las pacientes sin diabetes fue significativamente más alta que la de las pacientes con diabetes con un tamaño del efecto pequeño (Tablas 2 y 3 ). Al controlar estadísticamente el tiempo transcurrido desde 
el diagnóstico, usando ANCOVA, el efecto de la diabetes dejó de ser significativo, $\mathrm{F}_{(1,188)}=$ $2.07, p=.152, \eta_{p}^{2}=.01 ;$ por tanto, su efecto fue espurio.

Al comparar entre mujeres con tratamiento conservador y mastectomía, usando la prueba $\mathrm{t}$ de Student, o al comparar entre mujeres con tratamiento conservador, mastectomía y pendiente de operación, usando ANOVA, no se encontraron diferencias significativas en las medias de la puntuación total del BSI-18 y sus tres factores. No obstante, al comparar entre las mujeres mastectomizadas que deseaban o no conservar la mama, hubo diferencias significativas. Las medias de las mujeres que querían conservar la mama fueron más altas que las de quienes que no querían conservarla tanto en la puntuación total del BSI-18 como en sus tres factores. Los tamaños del efecto fueron grandes en BSI-18 y depresión, y medianos en los otros dos factores (Tablas 2 y 3). Al controlar estadísticamente el efecto del tiempo transcurrido desde el diagnóstico por ANCOVA, el efecto de desear o no conservar la mama siguió siendo significativo sobre la puntuación total del BSI-18, $\mathrm{F}_{(1,64)}=12.93, p$ $=.001, \eta_{\mathrm{p}}^{2}=.17 ;$ los síntomas vasovagales de ansiedad, $\mathrm{F}_{(1,64)}=8.05, p=.006, \eta_{\mathrm{p}}{ }^{2}=.11$; los síntomas de depresión, $\mathrm{F}_{(1,64)}=12.45, p=.001$, $\eta_{\mathrm{p}}{ }^{2}=.16, \mathrm{y}$ los síntomas simpáticos de ansiedad, $\mathrm{F}_{(1,64)}=7.58, p=.008, \eta_{\mathrm{p}}{ }^{2}=.11$. Igualmente, al controlar estadísticamente el efecto del tiempo transcurrido desde la cirugía por análisis de covarianza, el efecto de desear o no conservar la mama siguió siendo significativo sobre la puntuación total del BSI-18, $\mathrm{F}_{(1,63)}=12.18, p$ $=.001, \eta_{\mathrm{p}}{ }^{2}=.16 ;$ los síntomas vasovagales de ansiedad, $\mathrm{F}_{(1,63)}=7.88, p=.007, \eta_{\mathrm{p}}^{2}=.11 ; \operatorname{los}$ síntomas de depresión, $\mathrm{F}_{(1,63)}=11.18, p=.001$, $\eta_{\mathrm{p}}{ }^{2}=.15$, y los síntomas simpáticos de ansiedad, $\mathrm{F}_{(1,63)}=8.33, p=.005, \eta_{\mathrm{p}}^{2}=.12$.

Al comparar las medias en la puntuación total del BSI-18 y sus tres factores por tipos de tratamientos (cirugía, quimioterapia, radioterapia, inmunoterapia y terapia antihormonal), se hallaron diferencias significativas en ocho de las 20 comparaciones. Las mujeres que no han recibido quimioterapia tuvieron una media en síntomas de depresión significativamente más alta que las mujeres que sí la han recibido, con un tamaño mediano del efecto; a su vez, las mujeres que no han recibido quimioterapia tuvieron una media en síntomas simpáticos de ansiedad significativamente más alta que las mujeres que sí la han recibido, con un tamaño del efecto también mediano. Las mujeres que han recibido radioterapia tuvieron medias más altas en la puntuación total del BSI-18 y en el factor de síntomas simpáticos de ansiedad que las que no han recibido, con tamaños del efecto medianos. Las mujeres que han recibido terapia antihormonal tuvieron medias más altas en la puntuación total del BSI-18 y sus tres factores que las que no han recibido, con tamaños del efecto medianos en BSI-18 y síntomas simpáticos de ansiedad, y pequeños en los otros dos factores (Tablas 2 y 3 ). 
Tabla 2

Significación y tamaño del efecto en la comparación de medias en BSI-18 y sus tres factores por grupos de variables clínicas

\begin{tabular}{|c|c|c|c|c|c|c|}
\hline \multirow[b]{2}{*}{ Variables clínicas } & \multirow[b]{2}{*}{$\mathrm{n}$} & \multirow[b]{2}{*}{ Est. } & \multicolumn{4}{|l|}{ BSI-18 } \\
\hline & & & $\begin{array}{l}\text { Puntuación } \\
\text { total }\end{array}$ & $\begin{array}{l}\text { Síntomas } \\
\text { vasovagales } \\
\text { de ansiedad }\end{array}$ & $\begin{array}{l}\text { Síntomas } \\
\text { de depresión }\end{array}$ & $\begin{array}{l}\text { Síntomas } \\
\text { simpáticos } \\
\text { de ansiedad }\end{array}$ \\
\hline Tiempo desde diagnóstico & 195 & $r$ & $.142^{*}$ & $.084^{\mathrm{ns}}$ & $.113^{\text {ns }}$ & $.160^{*}$ \\
\hline Tiempo desde cirugía & 115 & $r$ & $.260^{* *}$ & $.072^{\text {ns }}$ & $.227^{*}$ & $.364^{* * *}$ \\
\hline Etapa & 191 & $r_{s}$ & $.086^{\mathrm{ns}}$ & $.067^{\mathrm{ns}}$ & $.060^{\text {ns }}$ & $.095^{\mathrm{ns}}$ \\
\hline Comorbilidad & 191 & $t$ & $0.967^{\text {ns }}$ & $0.271^{\mathrm{ns}}$ & $1.533^{\mathrm{ns}}$ & $0.528^{\text {ns }}$ \\
\hline (Sí vs. No) & & $g_{c}$ & 0.156 & 0.044 & 0.247 & 0.085 \\
\hline Hipertensión & 191 & $t$ & $-0.484^{\text {ns }}$ & $-0.800^{\text {ns }}$ & $0.259^{\text {ns }}$ & $-0.779^{n s}$ \\
\hline (Sí vs. No) & & $g_{c}$ & -0.087 & -0.145 & 0.047 & -0.141 \\
\hline Diabetes & 191 & $t$ & $-0.536^{n s}$ & $-0.838^{\text {ns }}$ & $0.767^{\text {ns }}$ & $-2.262^{*}$ \\
\hline (Sí vs. No) & & $g_{c}$ & -0.102 & -0.119 & 0.146 & -0.318 \\
\hline Antecedentes familiares & 191 & $t$ & $-1.263^{\mathrm{ns}}$ & $-1.155^{\mathrm{ns}}$ & $-0.493^{\text {ns }}$ & $-1.617^{\mathrm{ns}}$ \\
\hline (Sí vs. No) & & $g_{c}$ & -0.184 & -0.168 & -0.072 & -0.235 \\
\hline Antecedentes personales & 191 & $t$ & $-1.185^{\mathrm{ns}}$ & $-1.060^{\mathrm{ns}}$ & $-1.370^{\text {ns }}$ & $-0.511^{\mathrm{ns}}$ \\
\hline (Sí vs. No) & & $g_{c}$ & -0.317 & -0.284 & -0.367 & -0.137 \\
\hline TN (RE+RP+HER2) & 191 & $t$ & $0.731^{\mathrm{ns}}$ & $0.318^{\mathrm{ns}}$ & $0.502^{\mathrm{ns}}$ & $1.029^{\text {ns }}$ \\
\hline (Sí vs. No) & & $g_{c}$ & 0.119 & 0.052 & 0.082 & 0.168 \\
\hline Tipo de cirugía & 115 & $t$ & $0.911^{\mathrm{ns}}$ & $0.404^{\mathrm{ns}}$ & $0.632^{\text {ns }}$ & $1.299^{\mathrm{ns}}$ \\
\hline (C vs $M)$ & & $g_{c}$ & 0.172 & 0.076 & 0.119 & 0.246 \\
\hline Cirugía & 195 & $F$ & $0.617^{\text {ns }}$ & $0.110^{\text {ns }}$ & $0.340^{\text {ns }}$ & $1.164^{\mathrm{ns}}$ \\
\hline$(C, M$ y $P)$ & & $\eta$ & .080 & .034 & .059 & .109 \\
\hline Deseo conservar mama & 67 & $t$ & $3.523^{* * *}$ & $2.824^{* *}$ & $3.508^{* * *}$ & $2.679^{\star *}$ \\
\hline (en M Sí vs. No) & & $g_{c}$ & 0.861 & 0.689 & 0.856 & 0.654 \\
\hline Haber recibido cirugía & 195 & $t$ & $-0.543^{\text {ns }}$ & $-0.194^{\mathrm{ns}}$ & $-0.499^{n s}$ & $-0.665^{\mathrm{ns}}$ \\
\hline (CyN vs P) & & $g_{c}$ & -0.079 & -0.028 & -0.072 & -0.096 \\
\hline Quimioterapia & 191 & $t$ & $-2.030^{\mathrm{ns}}$ & $-1.212^{\mathrm{ns}}$ & $-2.195^{*}$ & $-2.215^{*}$ \\
\hline (Sí vs. No) & & $g_{c}$ & -0.834 & -0.540 & -0.770 & -0.765 \\
\hline Radioterapia & 191 & $t$ & $2.339^{*}$ & $1.583^{\mathrm{ns}}$ & $1.689^{\text {ns }}$ & $2.772^{\star \star}$ \\
\hline (Sí vs. No) & & $g_{c}$ & 0.518 & 0.292 & 0.372 & 0.645 \\
\hline Inmunoterapia & 191 & $t$ & $-0.5^{\mathrm{ns}}$ & $-0.877^{\mathrm{ns}}$ & $-0.788^{\text {ns }}$ & $0.407^{\text {ns }}$ \\
\hline (Sí vs. No) & & $g_{c}$ & -0.133 & -0.228 & -0.205 & 0.106 \\
\hline Terapia antihormonal & 191 & $t$ & $2.911^{* *}$ & $2.052^{*}$ & $2.034^{*}$ & $3.380^{* *}$ \\
\hline (Sí vs. No) & & $g_{c}$ & 0.691 & 0.489 & 0.461 & 0.797 \\
\hline
\end{tabular}

Nota. Cirugía: $\mathrm{C}=$ conservadora, $\mathrm{M}=$ mastectomía total y $\mathrm{P}=$ pendiente. Est. = estadístico: $r=$ correlación producto momento de Pearson, $t=$ valor del estadístico t de student para el contraste de la diferencia de medias de dos muestras independientes, gc =estadístico g corregido de sesgo de Hedges y Olkin (1985) para estimar el tamaño del efecto en los contrastes de medias por la prueba t de Student, $F=$ valor del estadístico $F$ de Fisher del análisis de varianza de una vía para $\mathrm{k}$ muestras independientes, $\eta=$ coeficiente eta para estimar el tamaño del efecto del estadístico F. Significación de la pruebas en un contraste bilateral: ns $=$ no significativa $p>.05,{ }^{*} p \leq .06,{ }^{* *} p \leq .01 \mathrm{y}{ }^{* * *} p \leq .001$. Fuente: elaborada por los autores. 
Tabla 3

Medias y diferencias medias en BSI-18 y sus tres factores por grupos diferenciales de variables clínicas

\begin{tabular}{|c|c|c|c|c|c|c|c|c|}
\hline \multirow[t]{2}{*}{ Variables } & \multicolumn{2}{|c|}{ PT } & \multicolumn{2}{|c|}{ SVA } & \multicolumn{2}{|c|}{ SDE } & \multicolumn{2}{|c|}{ SSA } \\
\hline & Sí & No & Sí & No & Sí & No & Sí & No \\
\hline \multirow{2}{*}{$\begin{array}{l}\text { Diabetes } \\
\text { comórbida }\end{array}$} & & & & & & & $\begin{array}{l}0.48 \\
{[0.34,} \\
0.63]\end{array}$ & $\begin{array}{l}0.71 \\
{[0.60,} \\
0.82]\end{array}$ \\
\hline & & & & & & & \multicolumn{2}{|c|}{$\begin{array}{l}-0.229 \\
{[-0.417,-0.024]}\end{array}$} \\
\hline \multirow{3}{*}{$\begin{array}{l}\text { Deseo de conservar } \\
\text { mama }\end{array}$} & $\begin{array}{l}0.95 \\
{[0.66} \\
1.25]\end{array}$ & \multirow[t]{2}{*}{$\begin{array}{l}0.38 \\
{[0.28} \\
0.48]\end{array}$} & $\begin{array}{l}0.88 \\
{[0.61} \\
1.16]\end{array}$ & $\begin{array}{l}0.41 \\
{[0.28} \\
0.54]\end{array}$ & $\begin{array}{l}1.10 \\
{[0.71,} \\
1.50]\end{array}$ & \multirow[t]{2}{*}{$\begin{array}{l}0.36 \\
{[0.22,} \\
0.53]\end{array}$} & $\begin{array}{l}0.87 \\
{[0.56} \\
1.19]\end{array}$ & \multirow[t]{2}{*}{$\begin{array}{l}0.37 \\
{[0.25,} \\
0.51]\end{array}$} \\
\hline & 0.569 & & \multicolumn{2}{|c|}{0.472} & 0.738 & & 0.496 & \\
\hline & \multicolumn{2}{|c|}{$[0.246,0.865]$} & \multicolumn{2}{|c|}{$[0.177,0.779]$} & \multicolumn{2}{|c|}{$[0.327,1.146]$} & \multicolumn{2}{|c|}{$\left[0.142,0.847^{\star}\right.$} \\
\hline \multirow{3}{*}{ Quimioterapia } & & & & & $\begin{array}{l}0.60 \\
{[0.49} \\
0.73]\end{array}$ & $\begin{array}{l}1.21 \\
{[0.68} \\
1.77]\end{array}$ & $\begin{array}{l}0.62 \\
{[0.52} \\
0.72]\end{array}$ & $\begin{array}{l}1.17 \\
{[0.73} \\
1.66]\end{array}$ \\
\hline & & & & & \multicolumn{2}{|c|}{-0.617} & \multicolumn{2}{|c|}{-0.543} \\
\hline & & & & & \multicolumn{2}{|c|}{$[-1.168,-0.099]$} & \multicolumn{2}{|c|}{$[-1.045,-0.075]$} \\
\hline \multirow{3}{*}{ Radioterapia } & $\begin{array}{l}0.95 \\
{[0.73} \\
1.19]\end{array}$ & $\begin{array}{l}0.63 \\
{[0.54} \\
0.72]\end{array}$ & & & & & $\begin{array}{l}1.04 \\
{[0.76} \\
{[1.34]}\end{array}$ & $\begin{array}{l}0.58 \\
{[0.48]} \\
0.70]\end{array}$ \\
\hline & 0.319 & & & & & & 0.453 & \\
\hline & \multicolumn{2}{|c|}{$[0.069,0.603]$} & & & & & \multicolumn{2}{|c|}{$[0.119,0.814]$} \\
\hline \multirow{3}{*}{ Terapia Antihormonal } & $\begin{array}{l}1.05 \\
{[0.78} \\
1.32]\end{array}$ & $\begin{array}{l}0.62 \\
{[0.54} \\
0.72]\end{array}$ & $\begin{array}{l}1.04 \\
{[0.75} \\
1.36]\end{array}$ & $\begin{array}{l}0.70 \\
{[0.61} \\
0.82]\end{array}$ & $\begin{array}{l}0.96 \\
{[0.64,} \\
1.32]\end{array}$ & $\begin{array}{l}0.59 \\
{[0.47} \\
0.73]\end{array}$ & $\begin{array}{l}1.13 \\
{[0.84} \\
1.43]\end{array}$ & $\begin{array}{l}0.58 \\
{[0.47} \\
0.69]\end{array}$ \\
\hline & 0.421 & & 0.337 & & 0.372 & & 0.554 & \\
\hline & \multicolumn{2}{|c|}{$[0.170,0.696]$} & \multicolumn{2}{|c|}{$[0.059,0.650]$} & \multicolumn{2}{|c|}{$[0.049,0.742]$} & \multicolumn{2}{|c|}{$[0.259,0.860]$} \\
\hline
\end{tabular}

Nota. Medias aritméticas y diferencias medias con su valor puntual y estimación por intervalo con un nivel de confianza al $95 \%$ por el método de muestreo repetitivo corregido de sesgo y acelerado con la simulación de 1,000 muestras aleatorias. PT = Puntuación total de BSI-18, SVA = síntomas vasovagales de ansiedad, SDE = síntomas depresivos y SSA = síntomas simpáticos de ansiedad. Fuente: elaborada por los autores.

Al controlar estadísticamente el efecto del tiempo transcurrido desde el diagnóstico por ANCOVA, el efecto de la quimioterapia sobre los síntomas de depresión siguió siendo significativo, $\mathrm{F}_{(1,188)}=9.01, p=.003, \eta_{\mathrm{p}}{ }^{2}=.05$. Lo mismo ocurrió con el efecto de la radioterapia sobre los síntomas simpáticos de ansiedad, $\mathrm{F}_{(1,188)}=6.41, p=.012, \eta_{\mathrm{p}}{ }^{2}=.03 \mathrm{y}$ el de la terapia antihormonal sobre la puntuación total del BSI$18, \mathrm{~F}_{(1,188)}=8.34, p=.004, \eta_{\mathrm{p}}{ }^{2}=.04$; los síntomas vasovagales de ansiedad, ${ }_{\mathrm{F}(1,188)}=4.48, p=.036$, $\eta_{\mathrm{p}}^{2}=.02$, y síntomas simpáticos de ansiedad, 
$\mathrm{F}_{(1,188)}=11.43, p=.001, \eta_{\mathrm{p}}^{2}=.06$. No obstante, el efecto de la radioterapia sobre la puntuación total del BSI-18 dejó de ser significativo, $\mathrm{F}_{(1,188)}$ $=3.51, p=.063, \eta_{\mathrm{p}}{ }^{2}=.02$, al igual que el efecto de la terapia antihormonal sobre los síntomas de depresión, $\mathrm{F}_{(1,188)}=3.31, p=.071, \eta_{\mathrm{p}}^{2}=.02$.

En los contrastes paramétricos (prueba $t$, ANOVA y ANCOVA), los intervalos corregidos de sesgo y acelerados con un nivel de confianza al 95\% confirmaron sus resultados; ya sea de no significación, al ser el límite inferior y superior de distinto signo; o de significación, al ser el límite inferior y superior del mismo signo. En la Tabla 3, se pueden ver las estimaciones por intervalo para las diferencias medias significativas en BSI-18 y sus tres factores al comparar entre casos y no casos definidos por cinco variables clínicas.

\section{Modelos de regresión logística binaria \\ Puntuación total en el BSI-18.}

En la muestra total, al usar como punto de corte el percentil 75, P75 $=0.89$, los correlatos fueron la radioterapia y terapia antihormonal. El modelo de regresión logística binaria presentó como único predictor la terapia antihormonal. Recibir terapia antihormonal actuó como un factor de riesgo de pertenecer al grupo de distrés. Su tamaño del efecto fue mediano, $O R=2.81$, intervalo de confianza (IC) del 95\% [1.28, 6.16].

Al emplear como punto de corte el percentil $90, \mathrm{P} 90=1.5$, los correlatos significativos fueron quimioterapia, radioterapia, terapia antihormonal y tiempo transcurrido desde el diagnóstico en la muestra total. El modelo presentó dos predictores: la quimioterapia y la terapia antihormonal. Recibir quimioterapia, $O R=0.25$, IC del 95\% [0.07, 0.85], actuó como factor protector de pertenecer al grupo de distrés. Su tamaño del efecto fue mediano, $1 / O R=4$. Por el contrario, recibir terapia antihormonal actuó como un factor de riesgo de pertenecer al grupo de distrés. Su tamaño del efecto fue mediano, $O R=3.69$, IC del 95\% [1.38, 9.82].

En la submuestra de 66 mujeres con mastectomía, al usar como punto de corte el percentil 75 , aparecieron como correlatos significativos la radioterapia, la terapia antihormonal y el deseo de conservar la mama. En el modelo de regresión logística, el deseo de conservar la mama fue el único predictor con un tamaño del efecto muy grande, $O R=10.40$, IC del 95\% [2.12, 51.03]. Al emplear como punto de corte el percentil 90, recibir quimioterapia, terapia antihormonal $\mathrm{y}$ deseo de conservar la mama fueron correlatos significativos. El modelo predictivo quedó integrado por la quimioterapia, que actuó como un factor protector de presentar distrés, con un tamaño del efecto muy grande, $O R=0.03$, IC del 95\% [0.002, 0.46], 1/OR = 31.25.

\section{Síntomas vasovagales de ansiedad.}

En la muestra total, al usar como punto de corte el percentil 75, P75 = 1, ninguna variable fue correlato significativo; por consiguiente, no se estimó ningún modelo. Al emplear como punto de corte el percentil 90, P90 = 1.6, el único correlato significativo fue la terapia antihormonal. Recibir terapia antihormonal fue un predictor significativo y actuó como un factor de riesgo de síntomas vasovagales de ansiedad con un tamaño de efecto mediano, $O R=3.74$, IC del 95\% [1.40, 9.98]. 
En la submuestra de mujeres con mastectomía, con el percentil 75 como punto de corte, la quimioterapia fue el único correlato significativo (sin usar la corrección de Yates en la prueba chi-cuadrado). Al calcular el modelo de regresión logística no tuvo un peso significativo. Al usar como punto de corte el percentil 90, la quimioterapia y deseo de conservar la mama fueron los correlatos significativos. El modelo predictivo quedó integrado por la quimioterapia, que actuó como un factor protector de tener síntomas vasovagales de ansiedad, con un tamaño del efecto muy grande, $O R=0.03$, IC del 95\% [0.002, 0.46], $1 / O R=31.25$.

\section{Síntomas de depresión.}

En la muestra total, al usar como punto de corte el percentil 75, $\mathrm{P} 75=0.67$, ninguna variable fue correlato significativo; por consiguiente, no se estimó ningún modelo. Al emplear como punto de corte el percentil 90, P90 = 2, la quimioterapia y tiempo transcurrido desde el diagnóstico resultaron correlatos significativos. El único predictor significativo fue recibir radioterapia, que actuó como un factor de riesgo de pertenecer al grupo de síntomas depresivos. Su tamaño del efecto fue mediano, $O R=3.12$, IC del 95\% [1.18, 8.23].

En la submuestra de mujeres con mastectomía, con el percentil 75 , no hubo ningún correlato significativo. Al usar como punto de corte el percentil 90, la terapia antihormonal fue el único correlato significativo. En el modelo predictivo su peso fue significativo y actuó como un factor de riesgo de tener síntomas de depresión, con un tamaño del efecto grande, $O R=4.63$, IC del $95 \%[1.53,13.98]$.

\section{Síntomas simpáticos de ansiedad.}

En la muestra total, usando como punto de corte el percentil $75, \mathrm{P} 75=0.83$, la radioterapia, la terapia antihormonal, la diabetes comórbida y el tiempo transcurrido desde el diagnóstico fueron correlatos significativos. El modelo en la muestra total quedó integrado por dos predictores: terapia antihormonal y radioterapia. Recibir terapia antihormonal actuó como un factor de riesgo de síntomas simpáticos de ansiedad con un tamaño del efecto mediano, $O R$ $=3.39, \mathrm{IC}$ del 95\% [1.37, 8.41]. También recibir radioterapia actuó como un factor de riesgo con un tamaño del efecto mediano, $O R=2.58$, IC del 95\% [1.09, 6.13]. Al emplear como punto de corte el percentil 90, $\mathrm{P} 90=1.5$, la quimioterapia, la radioterapia y la terapia antihormonal fueron correlatos significativos. El modelo retuvo un único predictor. Recibir terapia antihormonal actuó como un factor de riesgo de pertenecer al grupo de síntomas simpáticos de ansiedad. $\mathrm{Su}$ tamaño del efecto fue mediano, $O R=3.45$, IC del 95\% [1.31, 9.11].

En la submuestra de mujeres con mastectomía, con el punto de corte del percentil 75, hipertensión comórbida, recibir quimioterapia, radioterapia y terapia antihormonal fueron correlatos significativos. Al calcular los modelos de regresión logística, la terapia antihormonal actuó como un factor de riesgo con un tamaño del efecto muy grande, $O R=11.96$, IC del 95\% [3.53, 40.51]. Al usar como punto de corte el percentil 90, el deseo de conservar la mama, recibir quimioterapia, radioterapia y terapia antihormonal fueron los correlatos significativos. El 
modelo predictivo quedó integrado por la quimioterapia, que actuó como un factor protector, con un tamaño del efecto inmenso, $O R=0.02$, IC del 95\% [0.002, 0.34], 1/OR = 41.67.

\section{Discusión}

Se enunció como primer objetivo comprobar la relación de las variables sociodemográficas y clínicas con el BSI-18 y sus tres factores a efectos de aportar evidencias de validez concurrente para la escala y el nuevo modelo factorial definido por Miaja y Moral (2017). Además, se añadió un segundo objetivo, predecir el distrés con sus correlatos significativos. La expectativa era que la mayoría de las variables clínicas fueran correlatos y predictores del BSI-18 y sus tres factores (Hjörleifsdóttir et al., 2006; Reich et al., 2008), mientras que las variables sociodemográficas tuvieran menor peso, destacando entre ellas la edad (Champion et al., 2014).

Se confirmó el hecho de que las variables sociodemográficas tuvieran un menor peso que las clínicas. Las cuatro variables sociodemográficas resultaron no significativas, incluida la edad, como también reportaron Andreu et al. (2012) en España.

La independencia de la edad no puede atribuirse a un rango muy limitado en edades, ya que la media de edad de las participantes fue de 48 años, abarcando desde adultas jóvenes (con una mínima de 30 años) hasta adultas mayores (con una máxima de 79). Tampoco puede atribuirse a una distribución muy concentrada, ya que su perfil fue mesocúrtico y el coeficiente de variación fue de 20.4. Cabe mencionar que algunos estudios señalan que el efecto de la edad está mediado por variables clínicas y sociodemográficas tanto en la calidad de vida (Fang-Rong, Ming, Xia, Ya-Hong, Long-Shu, \& You-Guo, 2012) como en el estado emocional (Osborne, Elsworth, \& Hopper, 2003), por lo que su relación con el distrés resulta más bien espuria o no significativa en algunos estudios.

Las correlaciones de la escolaridad con el BSI-18 y sus tres factores en el presente estudio fueron negativas conforme a la expectativa de que un mayor grado de escolaridad se asociara a un menor nivel de distrés al implicar mayores recursos cognitivos para afrontar la situación de salud adversa (Miaja \& Moral, 2014). No obstante, la relación no fue significativa como en otras investigaciones (Andreu et al., 2012; Jacobsen et al., 2005; Kagee, Roomaney, \& Knoll, 2018).

Parece que estar casada, en comparación con estar soltera, disminuye el distrés por el apoyo del cónyuge (Budden et al., 2014; Fávez et al., 2015). La media en las puntuaciones en BSI-18 y sus tres factores fueron más bajas en mujeres casadas que en mujeres solteras, pero la diferencia no fue significativa ni aún al hacer las comparaciones por las pruebas de Tukey o de Fisher. La complejidad de la relación solo con el cónyuge y los hijos hace que este efecto no sea claramente reproducible de un estudio a otro, como revelan diversos reportes (Andreu et al., 2012; Jacobsen et al., 2005; Kagee et al., 2018).

Con relación al ingreso económico, podría cuestionarse si su falta de significación es atribuible a la confiabilidad de la información 
proporcionada por las participantes, ya que el estudio se realizó en mujeres atendidas en un hospital privado al que accedieron a través de un seguro dirigido a personas de bajos recursos. El seguro al que se hace referencia es el Seguro Popular, el cual es percibido por quienes no tienen acceso a mejores pólizas ofertadas por otras instituciones. Pueden ser beneficiarios el cónyuge, la pareja en unión libre, los padres mayores dependientes y los hijos dependientes de hasta 25 años o con discapacidad (Comisión Nacional de Protección Social en Salud, 2019). La concesión y retirada del Seguro Popular no depende del hospital, sino de instituciones públicas. Falsear la información sobre ingresos económicos al contestar la encuesta, la cual tenía garantizadalaconfidencialidaddelainformación, no tiene razón de ser. Además, la pregunta sobre el ingreso tenía la opción "desconozco/no deseo compartir la información”, que sería una salida para una persona ante la disyuntiva de ocultar información y solo cuatro mujeres eligieron esta opción.

La falta de significación del ingreso económico debe atribuirse a que es una variable con tamaño del efecto pequeño sobre el distrés (Carlson et al., 2004) o no significativo (Andreu et al., 2012; Jacobsen et al., 2005; Kagee et al., 2018), ya que el tratamiento en sus aspectos económicos, especialmente en el caso del cáncer de mama, es solventado por algún tipo de seguro o programa de beneficencia. Lo abrumador o estresante para estas mujeres son otros aspectos, como si van a sobrevivir y qué pasará con su familia en caso de morir (An et al., 2017).
En un estudio realizado en España, Rey et al. (2017) reportaron que las mujeres laboralmente inactivas y las amas de casa presentaban mayores niveles de ansiedad rasgo que las laboralmente activas en el momento del diagnóstico del cáncer de mama; no obstante, el estado laboral no afectó a la ansiedad estado ni a la calidad de vida. En el presente estudio, las medias más bajas en BSI-18 y sus tres factores se presentaron en mujeres jubiladas y las medias más altas en mujeres incapacitadas y trabajadoras activas sin diferencia significativa, ya sea en la comparación ómnibus (ANOVA) o en las comparaciones por pares (Tukey o Fisher). Cabe señalar que la edad fue estadísticamente equivalente entre las cinco categorías laborales comparadas. Este resultado es semejante al obtenido en el estudio de Andreu et al. (2012), en el que el estado laboral tampoco tuvo efecto sobre el distrés; asimismo, es concordante con el de Rey et al. (2017), ya que el distrés es una variable de estado y no una variable de rasgo.

En la presente investigación, entre las variables clínicas, destacaron el efecto de recibir terapia antihormonal y el deseo de conservar la mama; estas actuaron como factores de riesgo, conforme con las expectativas, con un tamaño del efecto mediano en los modelos predictivos la primera, y una fuerza de asociación moderada la segunda. Recibir terapia antihormonal predijo tanto presencia de distrés como de síntomas simpáticos y vasovagales de ansiedad y síntomas de depresión. No obstante, resultó un correlato espurio de depresión ante el efecto del tiempo transcurrido desde el diagnóstico. Cabe señalar 
que la terapia antihormonal tiene diversos efectos secundarios, entre ellos la inducción de la menopausia, lo que permite comprender su efecto potenciador del distrés (Ates et al., 2016).

También la radioterapia $y$ el tiempo transcurrido desde el diagnóstico actuaron como factores de riesgo. La radioterapia tuvo tamaños del efecto medianos sobre el factor de síntomas simpáticos de ansiedad y sobre el factor de depresión en los modelos de regresión logística binaria. El tiempo transcurrido desde el diagnóstico mostró una fuerza de asociación pequeña con el factor de síntomas simpáticos de ansiedad y con la puntuación total del BSI18, aunque no fue un predictor significativo. El hecho de que la radioterapia incrementara los casos de síntomas simpáticos de ansiedad se puede atribuir al malestar que genera el tratamiento y a la incertidumbre sobre su efecto (Luutonen, Vahlberg, Eloranta, Hyväri, \& Salminen, 2011).

El hecho de que el tiempo transcurrido desde el diagnóstico incrementara el distrés parece ir en contra de la afirmación previa de que el distrés desciende desde la etapa inicial del diagnóstico a las etapas de tratamiento. En la presente muestra de mujeres con cáncer de mama en tratamiento, la mayoría de ellas llevaba un año o menos de tratamiento ( $87 \%$ ) y las que rebasan el año estaban en tratamiento hormonal o paliativo (por recidivas o por ser diagnosticadas en una etapa más avanzada); entre estas mujeres se concentraron más los casos de distrés, lo que explica la relación encontrada. Por tanto, en un lapso entre el diagnóstico y el tratamiento breve (menos de un año), el porcentaje de finalización del tratamiento curativo fue más alto y el distrés descendió; pero una duración del tratamiento mayor a un año (mayor lapso de tiempo desde el diagnóstico) conllevó más tratamientos y más distrés, ya que esta situación estaba implicando un peor pronóstico (tratamiento paliativo) o tener receptores positivos hormonales que requerían terapia antihormonal, la cual induce menopausia y puede alterar el estado emocional (Kneece, 2017).

Al igual que el tiempo transcurrido desde el diagnóstico, el tiempo transcurrido desde la cirugía presentó asociación directa con la puntuación total y el factor de síntomas simpáticos de ansiedad. Además, también correlacionó con síntomas de depresión. Vahdaninia, Omidvari y Montazeri (2010), en un estudio prospectivo de mujeres iraníes con cáncer de mama, también hallaron que los niveles de ansiedad y depresión aumentaban con el tiempo; por el contrario, Andreu et al. (2012), en mujeres españolas con cáncer de mama, reportaron una disminución del distrés desde el diagnóstico preliminar al tratamiento.

En la presente muestra, entre las mujeres que han recibido tratamiento quirúrgico, aquellas con peor pronóstico o pronóstico reservado por receptores hormonales son las que llevan más tiempo en tratamiento; la mayoría de ellas han estando recibiendo terapia antihormonal, lo que explica esta correlación positiva. Vahdaninia et al. (2010) señalaban que los efectos secundarios a largo plazo del tratamiento podrían explicar la relación positiva entre distrés y tiempo 
transcurrido desde el diagnóstico. Cabría preguntarse si este aspecto también está contribuyendo al mayor distrés en las mujeres de este estudio, pero al no haberse evaluado los efectos secundarios del tratamiento no se pudo comprobar esta conjetura.

La quimioterapia se asoció a síntomas de depresión y simpáticos de ansiedad en la muestra total; además, fue un predictor significativo de casos de distrés, así como de síntomas vasovagales y simpáticos de ansiedad en la submuestra de mujeres con mastectomía. No obstante, actuó como factor protector, cuando se esperaba que fuese de riesgo como la terapia antihormonal y la radioterapia. Se podría conjeturar que el recibir quimioterapia disminuye los sentimientos de desesperanza y fantasías de muerte, y puede estar generando esperanza de sobrevivir. Asimismo, la quimioterapia fue predictor significativo en los modelos predictivos en la submuestra de mujeres mastectomizadas, pero no en la muestra total. En el momento de ser encuestadas, la mayoría de las mujeres mastectomizadas se encontraba en quimioterapia (dos tercios) y otras en terapia antihormonal (un cuarto) e inmunoterapia. El recibir quimioterapia tras la mastectomía disminuye el distrés, pero no así otras terapias. Tal vez el prestigio de esta terapia, a pesar de sus efectos secundarios, disminuye el temor a la recidiva del cáncer.

Entre los síntomas comórbidos se halló la diabetes como un correlato de síntomas simpáticos de ansiedad. Su presencia disminuía la queja de este tipo de síntomas de ansiedad, pero la diabetes finalmente resultó un correlato espurio ante el efecto del tiempo transcurrido desde el diagnóstico, de ahí que no es una variable relevante, aunque en algunos estudios surge como un correlato positivo de distrés (Holland et al, 2010). Cabría preguntarse si esto se debió a que la mayoría eran casos de diabetes tipo II no insulino-dependientes con buen control de síntomas. Con todo, el registro de la diabetes en los expedientes de las pacientes no permite responder a esta pregunta, y queda como una conjetura para futuros estudios.

Una limitación del estudio fue el uso de un muestreo no probabilístico. A favor de la muestra usada, en comparación con los estudios previos publicados, se tiene la homogeneidad en las características de sexo (todas mujeres), bajos recursos económicos, diagnóstico (cánceres de mama) y estar en fase de tratamiento, además de un tamaño muestral mayor que 200. Otra limitación es su diseño ex post facto transversal, por lo que no permite inferencias causales.

En conclusión, entre las 203 mujeres tratadas por cáncer de mama del presente estudio transversal, la puntuación total y los tres factores del BSI-18 son independientes de las variables sociodemográficas: edad, escolaridad, ingresos económicos familiares, situación laboral y estado civil. En esta muestra, recibir terapia antihormonal destaca como correlato y predictor entre las variables clínicas, seguida de radioterapia. Entre las 66 mujeres mastectomizadas, quimioterapia y deseo de conservar la mama fueron los correlatos y predictores más importantes. Estas 
variables actuaron como factores de riesgo de distrés, salvo la quimioterapia. Entre las mujeres mastectomizadas, recibir quimioterapia disminuye el distrés, cuando no es así con los otros tratamientos (hormonal e inmunológico). Mayores intervalos de tiempo transcurrido desde el diagnóstico y desde la cirugía se asocian a más distrés, pero su fuerza de asociación es pequeña y ambas variables no resultan predictores significativos en los modelos de regresión. Síntomas comórbidos de diabetes se asocian a síntomas simpáticos de ansiedad, con fuerza de asociación pequeña y sin ser un predictor significativo, pero hipertensión comórbida $\mathrm{y}$ antecedentes personales y familiares de cáncer resultan independientes del distrés.

Se sugiere confirmar el hallazgo de que la quimioterapia actúa como un factor protector de distrés en mujeres con mastectomía, e indagar su porqué mediante un enfoque cualitativo. Se hipotetiza que esta terapia debe reducir la expectativa de recidiva entre estas mujeres y proporcionar una mayor confianza en la cura. Asimismo, se sugiere un mayor apoyo psicológico para las mujeres que reciben terapia antihormonal y radioterapia, especialmente considerando que a más tiempo de tratamiento se incrementa el distrés (casos más complejos o con peor pronóstico). Las intervenciones enfocadas exclusivamente a reducir el distrés no requieren establecer diferencias o controles en relación con la edad, estado civil, escolaridad, estado laboral e ingreso económico, pues estas variables no tienen efecto sobre el distrés o su efecto es mínimo.

\section{Referencias}

American Psychological Association. (2017). Ethical principles of psychologists and code of conduct. Washington, DC, EUA: American Psychological Association Press.

An, E., Wennberg, E., Nissim, R., Lo, C., Hales, S., \& Rodin, G. (2017). Death talk and relief of death-related distress in patients with advanced cancer. BMJ Supportive \& Palliative Care, 7, 1-12. https://doi. org/10.1136/bmjspcare-2016-001277

Andreu, Y., Galdón, M. J., Durá, E., Martínez, M., Pérez, S., \& Murgui, S. (2012). A longitudinal study of psychosocial distress in breast cancer: Prevalence and risk factors. Psychology \& Health, 27, 72-87. http://doi.or $\mathrm{g} / 10.1080 / 08870446.2010 .542814$

Ates, O., Soylu, C., Babacan, T., Sarici, F., Kertmen, N., Allen, D., \& Altundag, K. (2016). Assessment of psychosocial factors and distress in women having adjuvant endocrine therapy for breast cancer: the relationship among emotional distress and patient and treatment-related factors. SpringerPlus, 5, 486. http://doi.org/10.1186/ s40064-016-2136-2

Budden, L. M. Hayes, B. A., \& Buettner, P. G. (2014). Women's decision satisfaction and psychological distress following early breast cancer treatment: a treatment decision support role for nurses. International Journal of Nursing Practice, 20, 8-16. http://doi. org/10.1111/ijn.12243

Carlson, L. E., Angen, M., Cullum, J., Goodey, E., Koopmans, J., Lamont, L., \& Bultz, B. 
D. (2004). High levels of untreated distress and fatigue in cancer patients. British Journal of Cancer, 90, 2297-2304. http://doi. org/10.1038/sj.bjc. 6601887

Champion, V. L., Wagner, L. I., Monahan, P. O., Daggy, J., Smith, L., Cohee, A., \& Sledge, G. W. (2014). Comparison of younger and older breast cancer survivors and age-matched controls on specific and overall quality of life domains. Cancer, 120, 2237-2246. http://doi. org/10.1002/cncr.28737

Chinn, S. (2000). A simple method for converting an odds ratio to effect size for use in metaanalysis. Statistics in Medicine, 19(22), 3127-3131. http://dx.doi.org/10.1002/10970258(20001130)19:223.3.CO;2-D

Cohen, J. (1988). Statistical power analysis for the behavioral sciences. 2da edición. Hillsdale, EUA: Lawrence Erlbaum Associates.

Comisión Nacional de Protección Social en Salud. (2019). Fondo de protección contra gastos catastróficos. Recuperado de: https://www.gob.mx/salud/seguropopular/ documentos/fondo-de-proteccion-contragastos-catastroficos

Derogatis, L. R. (2001). Brief Symptom Inventory (BSI)-18. Administration, scoring and procedures manual. Minneapolis, EUA: NCS Pearson. https://doi.org/10.1037/t07502-000

Derogatis, L. R. (2013). BSI-18. Inventario Breve de 18 Síntomas. Valencia, España: Universidad de Valencia, SGS Tecnos, S.A. \& el Dpartamento I+D Pearson Clinical \& Talent Assessment.
Enríquez, H., Rodríguez, J. T., \& Schneider, R. E. (2010). Síndrome de intestino irritable y otros trastornos relacionados. Fundamentos biopsicosociales. Ciudad de México, México: Editorial Médica Panamericana.

Fang-Rong, S., Ming, L., Xia, Z., Ya-Hong, F., Long-Shu, Z., \& You-Guo, C. (2012). Health-related quality of life among breast cancer patients and its influencing factor in a Chinese population. Asian Pacific Journal of Cancer Prevention, 13, 3747-3750. http:// doi.org/10.7314/APJCP.2012.13.8.3747

Fávez, N., Notari, S. C., Charvoz, L., Notari, L., Ghisletta, P., Ruedin, B. P., \& Delaloye, J. F. (2015). Distress and body image disturbances in women with breast cancer in the immediate postsurgical period: The influence of attachment insecurity. Journal of Health Psychology, 21, 2994-3003. http:// doi.org/10.1177/1359105315589802

Hedges, L. V., \& Olkin, I. (1985). Statistical methods for meta-analysis. San Diego, EUA: Academic Press.

Hernández, M., Cruzado, J. A., Prado, M. C., Rodríguez, E., Hernández, C., González, M. A., \& Martín, J. C. (2012). Salud mental y malestar emocional en pacientes con cáncer. Psicooncología, 9(2-3), 299-316. http://doi. org/10.5209/rev_PSIC.2013.v9.n2-3.40895

Hjörleifsdóttir, E., Hallberg, I. R., Bolmsjö, I. A., \& Gunnarsdóttir, E. D. (2006). Distress and coping in cancer patients: feasibility of the Icelandic version of BSI 18 and the WOC-CA questionnaires. European Journal of Cancer Care, 15, 80-89. http://doi. $\operatorname{org} / 10.1111 /$ j.1365-2354.2005.00620.x 
Holland, J. C., Andersen, B., Breitbart, W. S., Compas, B., Dudley, M. M., Fleishman, S., \& Zevon, M. A. (2010). Distress management: Clinical practice guidelines in oncology. Journal of National Comprehensive Cancer Network, 8, 448-485. http://doi.org/10.6004/ jnccn.2010.0034

Jacobsen, P. B., Donovan, K. A., Trask, P. C., Fleishman, S. B., Zabora, J., Baker, F., \& Holland, J. C. (2005). Screening for psychologic distress in ambulatory cancer patients. Cancer, 103(7), 1494-1502. https:// doi.org/10.1002/cncr.20940

Kagan, A. R., \& Levi, L. (1975). Health and environmental-psychosocial stimuli: A review. En L. Levi (Ed.), Society, stress and disease (vol. 2, 241-260). Londres, Inglaterra: Oxford University Press.

Kagee, A., Roomaney, R., \& Knoll, N. (2018). Psychosocial predictors of distress and depression among South African breast cancer patients. Psycho-Oncology, 27(3), 908-914. https://doi.org/10.1002/pon.4589

Kankainen, A., Taskinen, S., \& Oja, H. (2007). Tests of multinormality based on location vectors and scatter matrices. Statistical Methods and Applications, 16(3), 357-379. https://doi.org/10.1007/s10260-007-0045-9.

Kneece, J. (2017) Breast cancer treatment handbook: Understanding the disease, treatments, emotions, and recovery from breast cancer. 9a edición. Nueva York, EUA: Springer.

Luutonen, S., Vahlberg, T., Eloranta, S., Hyväri, H., \& Salminen, E. (2011). Breast cancer patients receiving postoperative radiotherapy: distress, depressive symptoms and unmet needs of psychosocial support. Radiotherapy and Oncology, 100, 299-303. http://doi.org/10.1016/j.radonc.2011.01.014. Miaja, M., \& Moral J. (2014). Predicción de las respuestas psicológicas del duelo ante la pérdida de salud por variables de personalidad, religiosidad, sociodemográficas, y existenciales. Salud $y$ Sociedad, 5(3), 254-272. https://doi. org/10.22199/S07187475.2014.0003.00002

Miaja, M., \& Moral, J. (2017). Validación del Inventario Breve de Síntomas (BSI-18) en mujeres mexicanas diagnosticadas con cáncer de mama. Psicooncología, 14, 307324. http://doi.org/10.5209/PSIC.57088

Ojike, N., Sowers, J. R., Seixas, A., Ravenell, J., Rodriguez-Figueroa, G., Awadallah, M., \& McFarlane, S. I. (2016). Psychological distress and hypertension: Results from the National Health Interview Survey for 20042013. Cardiorenal Medicine, 6(3), 198-208. http://doi.org/10.1159/000443933

Osborne, R. H., Elsworth, G. R., \& Hopper, J. L. (2003) Age-specific norms and determinants of anxiety and depression in 731 women with breast cancer recruited through a populationbased cancer registry. European Journal of Cancer, 39, 755-762. http://doi.org/10.1016/ S0959-8049(02)00814-6

Owens, A. P., Low, D. A., Iodice, V., Critchley, H. D., \& Mathis, C. J. (2017). The genesis and presentation of anxiety in disorders of autonomic overexcitation. Autonomic 
Neuroscience: Basic and Clinical, 203, 81-87. http://doi.org/10.1016/j.autneu.2016.10.004

Reich, M., Lesur, A., \& Perdrizet-Chevallier, C. (2008). Depression quality of life and breast cancer: A review of the literature. Breast Cancer Research and Treatment, 110, 9-17. http://doi.org/10.1007/s10549-007-9706-5

Rey, R., Fernández, S., Garea, C., Pillado, M. T., Barreiro, V., \& Martín, C. (2017). Calidad de vida, ansiedad antes y después del tratamiento en mujeres con cáncer de mama. Revista Latino-Americana de Enfermagem, 25, e2958. https://dx.doi.org/10.1590/15188345.2258.2958

Sawilowsky, S. (2009). New effect size rules of thumb. Journal of Modern Applied Statistical Methods, 8(2), 467-474. https:// doi.org/10.22237/jmasm/1257035100

Segrin, C., \& Badger, T. A. (2014). Psychological and physical distress are interdependent in breast cancer survivors and their partners. Psychology, Health \& Medicine, 19, 716723. http://doi.org/10.1080/13548506.2013. 871304

Simon, E., Meuret, A. E., \& Ritz, T. (2017). Sympathetic and parasympathetic cardiac responses to phobia-relevant and disgustspecific emotion provocation in bloodinjection-injury phobia with and without fainting history. Psychophysiology, 54, 15121527. http://doi.org/10.1111/psyp.12900

Vahdaninia, M., Omidvari, S., \& Montazeri A. (2010). What do predict anxiety and depression in breast cancer patients? A follow-up study. Social Psychiatry and
Psychiatric Epidemiology, 45, 355-361. http://doi.org/10.1007/s00127-009-0068-7

Wolfe, F., Brähler, E., Hinz, A., \& Häuser, W. (2013). Fibromyalgia prevalence, somatic symptom reporting, and the dimensionality of polysymptomatic distress: Results from a survey of the general population. Arthritis Care \& Research, 65, 777-785. http://doi. org/10.1002/acr.21931

Zabora, J., Brintzenhofeszoc, K., Jacobsen, P., Curbow, B., Piantadosi, S., Hooker, C., \& Derogatis, L. (2001). A new psychosocial screening instrument for use with cancer patients. Psychosomatics, 42, 241-246. http:// doi.org/10.1176/appi.psy.42.3.241.

Recibido: 27/05/2019

Revisado: 28/06/2019

Aceptado: 26/07/2019 